\title{
Diagnostic potential of a serological assay for the diagnosis of ulcerans disease based on the putative Mycobacterium ulcerans toxin
}

\author{
BRENDA ROBERTS and R. G. HIRST \\ Department of Microbiology and Immunology, James Cook University of North Queensland, Townsville, \\ Queensland 4811, Australia
}

\begin{abstract}
Mycobacterium ulcerans infection is an important and potentially disfiguring disease of man. A rapid diagnostic assay for detection of this organism is required urgently. Serological assays require a species-specific protein to ensure a high level of specificity and thus reduce the occurrence of false positive results. As $M$. ulcerans had been reported to produce a unique cytotoxin, it was thought that this would provide an ideal antigen on which to base a serological assay for detection of $M$. ulcerans during infection. Crude culture filtrates, prepared by previously documented methods, were assayed for toxic activity by in-vitro cytotoxicity assays and in-vivo mouse footpad assays. To evaluate the uniqueness of the cytotoxic factor, other species of mycobacteria were also assayed. Analysis of these assays showed that similar biological activity is present in various other mycobacterial species. Furthermore, it was possible to neutralise this activity in all species tested with a polyclonal antiserum raised against $M$. ulcerans. As the cytotoxic factor was found not to be specific to $M$. ulcerans, it is unlikely that a serological assay based on such a molecule will be of use.
\end{abstract}

\section{Introduction}

Interest in the genus Mycobacterium has resulted primarily because of the clinical implications of $M$. tuberculosis infection. However, this species is only one of many in the genus that are of clinical importance. While the majority of the now nearly 50 listed mycobacterial species live as environmental saprophytes, many have been implicated in causing disease in man and domestic livestock.

M. ulcerans was first described in 1948 in Bairnsdale, a temperate region of Australia, as being the causative agent of progressive, superficial skin ulcers [1]. This disease is now known to occur in many tropical regions of the world, including North Queensland.

Although many years have elapsed since the disease was first reported, no rapid diagnostic assay has yet been developed. The diagnosis of ulcerans disease relies primarily upon the isolation and culture of the causative organisms. This procedure may take several months, during which time the size of the ulcer

Received 3 July 1996; revised version accepted 16 Sept. 1996

Corresponding author: Dr B. Roberts. increases substantially. Because of the poor response to chemotherapy, the only effective treatment involves wide surgical excision of the lesion and subsequent skin grafting. With the progressive invasion by the organism, up to one-third of a limb may become ulcerated before treatment commences, thus requiring major surgery and resulting in severe physical and psychological trauma to the patient. An early diagnosis would result in minimal surgical involvement by enabling treatment to commence while the ulcer was still in its initial stages.

M. ulcerans has been reported to elaborate an exotoxin [2-4], a feature unique within the genus Mycobacterium. Therefore, this putative toxin could be an ideal antigen on which to base a diagnostic enzyme-linked immunosorbent assay (ELISA). In the present study, the feasibility of developing such an assay was investigated.

\section{Materials and methods}

\section{Bacterial strains and growth conditions}

A total of eight strains of $M$. ulcerans was originally obtained from Ms Z. Blacklock, Microbiology and Pathology Laboratory, Queensland Health, Brisbane, 
Australia. These strains had been isolated from patients with active $M$. ulcerans infection and inoculated on to Lowenstein-Jensen media. They were subcultured in Dubos broth base supplemented with sheep serum $5.0 \%$ and incubated at $30^{\circ} \mathrm{C}$ with constant agitation. Mycobacterial species other than M. ulcerans were supplied as freeze-dried preparations by Oonoonba Veterinary Laboratory, DPI, Townsville, Australia. The species tested included $M$. smegmatis, M. phlei, $M$. fortuitum and $M$. chelonei. The bacterial pellets were rehydrated in $1 \mathrm{ml}$ of phosphate-buffered saline (PBS), $\mathrm{pH}$ 7.2. These suspensions were used to inoculate both Middlebrooks agar slopes (Difco, Sydney) and nutrient agar plates (Oxoid, Sydney). Once confluent growth of the organisms had been observed on solid medium, they were used to inoculate broth cultures. Liquid cultures of each species were grown in $200-\mathrm{ml}$ volumes of Dubos broth containing sheep serum 5\%. These cultures were incubated at $37^{\circ} \mathrm{C}$ for 3 days, with a magnetic stirrer and $2-\mathrm{cm}$ 'flea' to agitate and aerate the cultures continually.

\section{Toxin production}

After a 10 -week incubation period for M. ulcerans, and 3 days for the remainder of the mycobacterial species, the cultures were tested for sterility by streaking on nutrient agar and staining for acid-fast bacilli with the Ziehl-Neelsen stain. When quality was assured, each culture was centrifuged for $1 \mathrm{~h}$ at $12000 \mathrm{~g}$, and the supernate was filtered through a $0.45-\mu \mathrm{m}$ membrane. The resultant culture filtrate was concentrated to onetenth the original volume with an Amicon stirred cell concentrator fitted with a $10000 \mathrm{MW}$ cut-off filter (YM10) (Amicon, MA, USA), and then dialysed against three changes of PBS, pH 7.2, over $24 \mathrm{~h}$. The resultant preparation was designated the crude culture filtrate $(\mathrm{CCF})$.

\section{Polyacrylamide gel electrophoresis}

Culture filtrates of the mycobacterial species were prepared as described above, and the protein concentration was determined by the Pierce BCA method. Electrophoresis was performed as described earlier [5]. Both denaturing and native polyacrylamide gels were used during the analysis. The preparation and running conditions for both were identical, with the exception of the omission of sodium dodecyl sulphate (SDS) and 2-mercaptoethanol from all buffers used for native PAGE. After electrophoresis, the polyacrylamide gels were stained with either Coomassie Blue or silver stain.

\section{In-vivo toxicity assay}

The in-vivo activity of each mycobacterial species was determined by inoculating the right hind leg footpad of $\mathrm{BALB} / \mathrm{c}$ mice with $0.025 \mathrm{ml}$ of CCF. Control groups received either Dubos broth or PBS $\mathrm{pH}$ 7.2. Footpad thickness in both the treated and untreated feet of each mouse was measured with an Oditest micrometer. These measurements were performed 'double blind', with the specific inoculum used for the mouse unknown to the micrometer operator, and the face of the instrument visible only to a second person recording the values. Readings were taken four times daily for $72 \mathrm{~h}$, with 10 replicate readings taken from both feet of each mouse at every reading.

Footpad swelling was assessed by subtracting the average of the readings taken from the untreated foot $\left(f_{u}\right)$, from the average of the readings taken from the inoculated foot $\left(f_{t}\right)$, for each mouse in the trial. To determine if differences in the degree of swelling between each group of mice was statistically significant, a multifactorial analysis of variance (ANOVA) was applied to the data. Analysis involved univariate repeated measures analysis of variance. The response variable was the difference in swelling between $f_{t}$ and $f_{u}$. The repeated measures factor was time, and the between groups factor was treatments.

\section{In-vitro cytoxicity assay}

Suspensions of L-929 cells were maintained in logarithmic growth in CSL tissue culture medium (Commonwealth Serum Laboratories, Parkville) containing fetal calf serum (FCS) $10 \%$ and antibiotics (penicillin, $100 \mathrm{U} / \mathrm{ml}$; streptomycin, $100 \mu \mathrm{g} / \mathrm{ml}$ ). Semiconfluent monolayers were prepared by adding $5.0 \times 10^{5}$ cells/flask in $10 \mathrm{ml}$ of culture medium. Once confluent, the cells were harvested and used to inoculate 96-well tissue culture trays (Nunc) with $100 \mu \mathrm{l}$ of suspension/well. Once these monolayers had become semi-confluent, $100 \mu \mathrm{l}$ of the CCF preparations were added and titrated across the wells. Control wells were treated with either Dubos broth or CSL tissue culture medium. Protein concentrations of both the CCF and the Dubos broth were standardised to $10 \mathrm{mg} / \mathrm{ml}$ in the first wells. The trays were incubated at $37^{\circ} \mathrm{C}$ with $\mathrm{CO}_{2} 5 \%$ for $48 \mathrm{~h}$, at which time they were examined for evidence of cytopathic effects (CPE).

\section{Toxin neutralisation assay}

A polyclonal antiserum raised in a rabbit against $M$. ulcerans CCF was tested for its ability to neutralise the toxic factor present in the culture filtrate preparations. The following samples were prepared for testing: a 1$\mathrm{ml}$ sample of CCF from each mycobacterial species was incubated with $0.01 \mathrm{ml}$ of immune rabbit serum at $37^{\circ} \mathrm{C}$ for $30 \mathrm{~min}$, and a $1-\mathrm{ml}$ sample of Dubos broth containing sheep serum $5 \%$ was incubated at $37^{\circ} \mathrm{C}$ for $30 \mathrm{~min}$ with $0.01 \mathrm{ml}$ of immune rabbit serum. Untreated CCF preparations from each of the five species were used as the positive controls. These samples were tested for toxic activity by the mouse footpad assay as described above.

Data obtained from the neutralisation assay were 
analysed with the statistics program SPSS for MS Windows, Version 6.0. A total of 10 replicate readings was taken from each footpad at each time point for the duration of the trial. These were averaged, and $f_{u}$ was subtracted from $f_{t}$. In all, two different treatments were crossed with five different antigens, each having 10 replicate measurements at each timepoint. To determine if differences in the degree of footpad swelling between each group were statistically significant, the data were subjected to a factorial ANOVA.

\section{Results}

Results of electrophoretic analyses

A protein concentration of $0.05 \mathrm{mg}$ in CCF was found to give optimal results by both SDS and native PAGE. Each of the mycobacterial CCFs produced essentially identical protein profiles with both electrophoretic techniques, as shown in Figs. 1 and 2. When SDSpolyacrylamide gels were stained with silver stain, several bands not previously visible when stained with Coomassie Blue, were observed. However, the majority of bands remained common between all species tested.

\section{Results of in-vivo toxicity assay}

The data obtained from the mouse footpad assay were analysed by Tukey's multifactorial ANOVA, and the results are presented in Fig. 3. No significant interaction was found between time and treatments $\left(F_{(12)}=1.80, p=0.0555\right)$, thus any differences due to treatments were constant over time. Overall, there were differences between the test and the control groups $\left(F_{(5)}=9.59, \quad p=0.0007\right)$; however, there were no

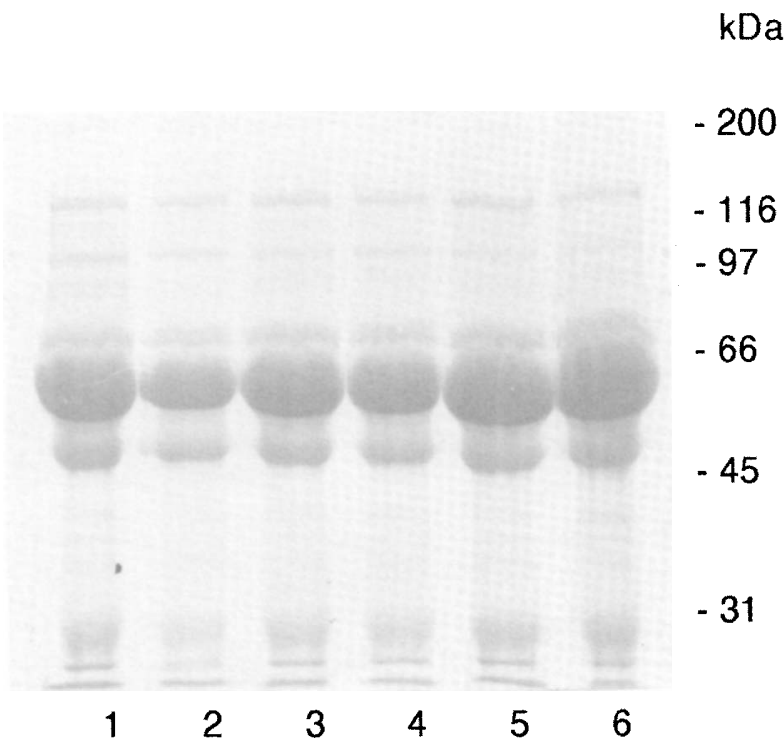

Fig. 1. Protein profiles of mycobacterial crude culture filtrates $(50 \mu \mathrm{g} / \mathrm{ml}$ protein $)$ resolved by SDS-PAGE. Lanes: 1, Dubos broth containing sheep serum 5\%; 2, M. chelonei; 3, M. fortuitum; 4, M. phlei; 5, M. smegmatis; 6, M. ulcerans.

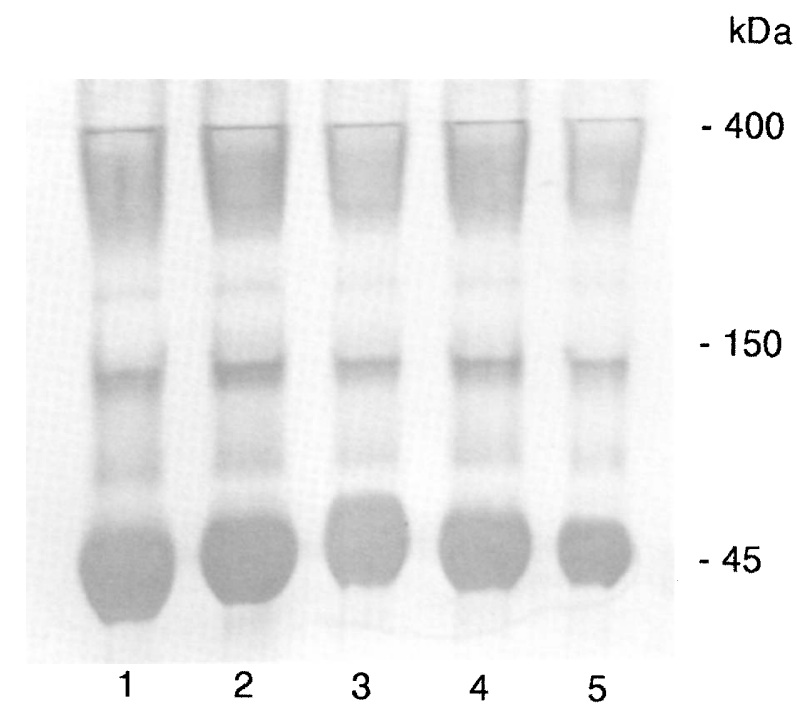

Fig. 2. Protein profiles of mycobacterial crude culture filtrates $(50 \mu \mathrm{g} / \mathrm{ml})$ resolved by native PAGE. Lanes: 1, $M$. chelonei; 2, M. fortuitum; 3, M. phlei; 4, M. smegmatis; 5, M. ulcerans.

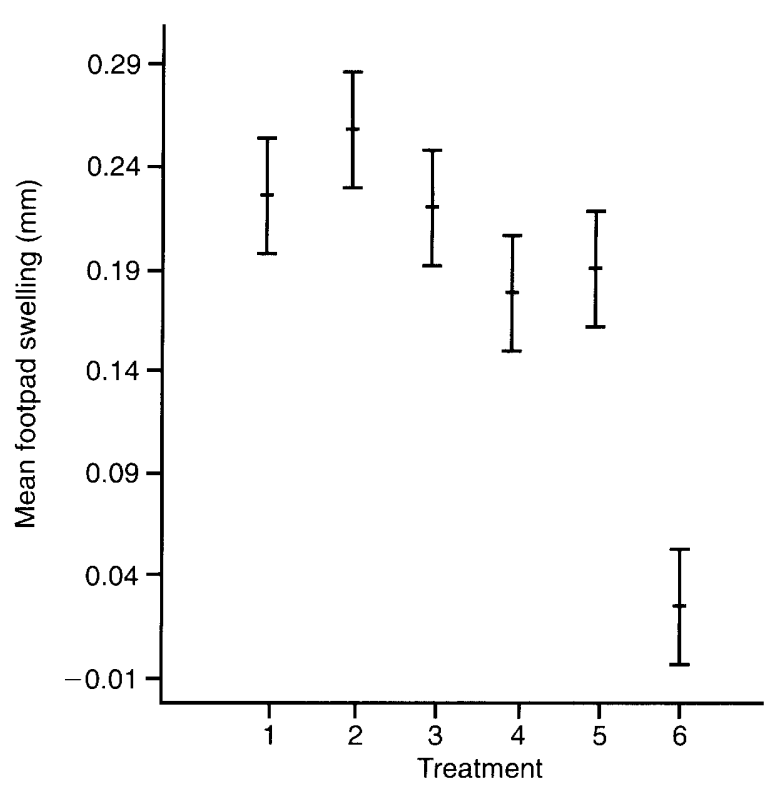

Fig. 3. Mean degree of footpad swelling, showing $95 \%$ confidence intervals, of mouse footpads in response to an injection of $0.025 \mathrm{ml}$ of mycobacterial crude culture filtrate. Treatments: 1, $M$. fortuitum; 2, M. ulcerans fortuitum; 3, M. smegmatis; 4, M. chelonei; 5, M. phlei; 6, Dubos broth containing sheep serum $5 \%$.

differences between the test groups $\left(\mathrm{F}_{(50)}=1.06\right.$, $\mathrm{p}=0.3782$ ).

\section{Results of in-vitro cytotoxicity assay}

The system of scoring used to determine the relative degree of CPE occurring in monolayers of L-929 cells $48 \mathrm{~h}$ after treatment is described in Table 1 . The degree of CPE was determined by the percentage of ovoid or detached cells, or cell showing complete autolysis. The results of the cytotoxicity assay presented in Fig. 4 
Table 1. Scoring system used to determine relative degree off CPE in L-929 cell monolayers as measured $48 \mathrm{~h}$ after the addition of sample

\begin{tabular}{ll}
\hline Score & Degree of CPE \\
\hline 0 & None \\
$1+$ & $<30 \%$ \\
$2+$ & $30-60 \%$ \\
$3+$ & $60-80 \%$ \\
$4+$ & $>80 \%$ \\
\hline
\end{tabular}

indicate that all mycobacterial species investigated produced a similar degree of CPE when tested on L929 cell monolayers. The end-point for $\mathrm{CPE}$ was between 20 and $40 \mu \mathrm{g}$ of protein $/ \mathrm{ml}$ of $\mathrm{CCF}$ for all Mycobacterium species tested. Fig. 5a shows the typical cytopathic effects observed when the L-929 monolayers were exposed to mycobacterial CCF. This can be compared to the appearance of untreated cells shown in Fig. 5 b.

\section{Results of toxin neutralisation assay}

The data obtained from this assay were analysed by a factorial ANOVA, and the results are presented in Fig. 6. The data were subsequently analysed at timepoints 4,28 and $68 \mathrm{~h}$ after inoculation to determine if any differences were significant. These points were chosen as being representative of differences at the beginning, middle and end of the trial. At each of these timepoints, no significant interaction was found between treatment and antigens, and no variation within antigen. The results presented in Table 2 show that there was no significant difference between treatments at 4 or $68 \mathrm{~h}$ after inoculation. However, at $28 \mathrm{~h}$ after inoculation, the difference was shown to be statistically significant $(F=63.78, p<0.001)$.

\section{Discussion}

Fractions in the culture filtrate of $M$. ulcerans found to exhibit toxic activity [2], were reported to have a mol. wt of $\geqslant 100 \mathrm{kDa}$. The proteins in the CCF of $M$. ulcerans were separated by PAGE and compared to those of other mycobacterial species in the hope of discovering a protein unique to $M$. ulcerans in the appropriate size range. Therefore, it was surprising to observe almost identical protein profiles from all the mycobacterial species tested, with both SDS and native PAGE. To investigate the possibility that the toxin molecule was not visualised due to a concentration factor, the resolved CCF proteins were silver stained. This technique is known to exhibit a higher degree of sensitivity than the standard Coomassie Blue stain for detecting proteins present in low concentrations. However, almost identical protein profiles were again obtained for all the mycobacterial species tested.

The high degree of similarity in the protein profiles in each mycobacterial CCF led to the speculation that the biologically active factor present in the CCF of $M$. ulcerans may also be present in that of other mycobacterial species. When the biological activity of all culture filtrates was investigated in both the invivo mouse footpad assay and the in-vitro cytotoxicity assay, this was found to be the case. The degree of footpad swelling for each of the CCFs did not differ significantly, but were all significantly different to the Dubos broth control. Similarly, all L-929 monolayers contained cells that displayed a range of cytopathic effects including margination of nuclear material, pyknotic nuclei and autolysis.

Therefore, it appears that the biological activity observed in $M$. ulcerans culture filtrate may be found also in filtrates of various other mycobacterial species.

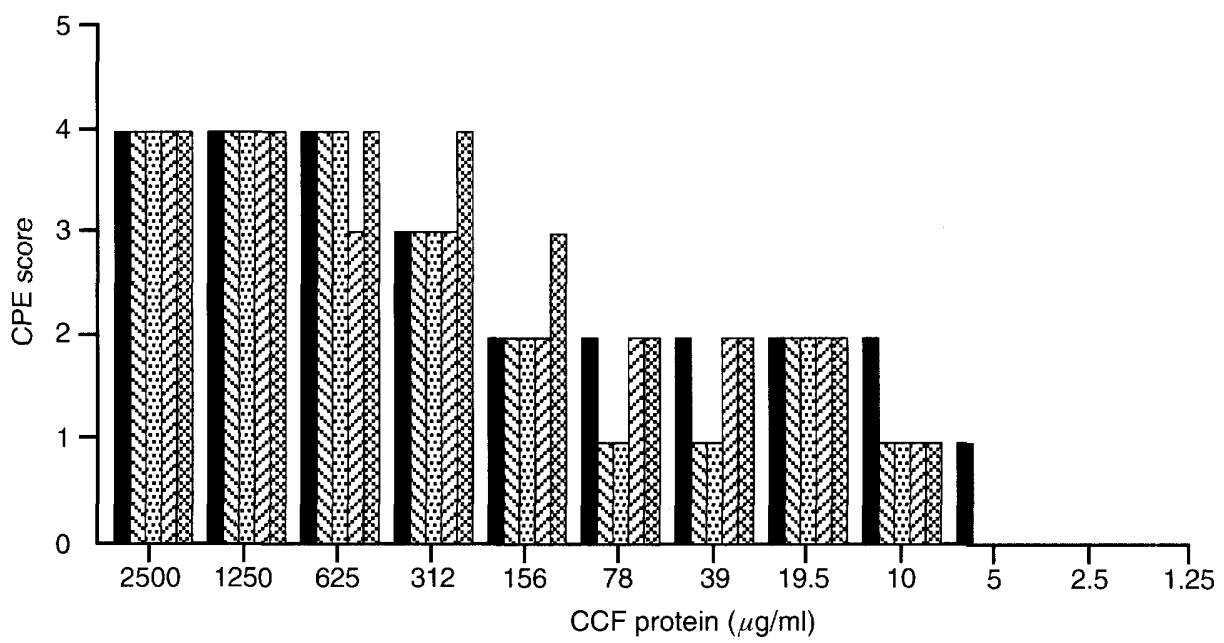

Fig. 4. Degree of cytopathic effect (as described in Table 1) displayed by monolayers of L-929 cells following exposure to a range of mycobacterial crude culture filtrate concentrations at $37^{\circ} \mathrm{C}$ over $48 \mathrm{~h}$. $\mathbf{\square}, M$. ulcerans; $\not, M$. fortuitum; $\mathbf{\mathrm { S }}$, M. smegmatis; 要, M. phlei; , M. chelonei. 

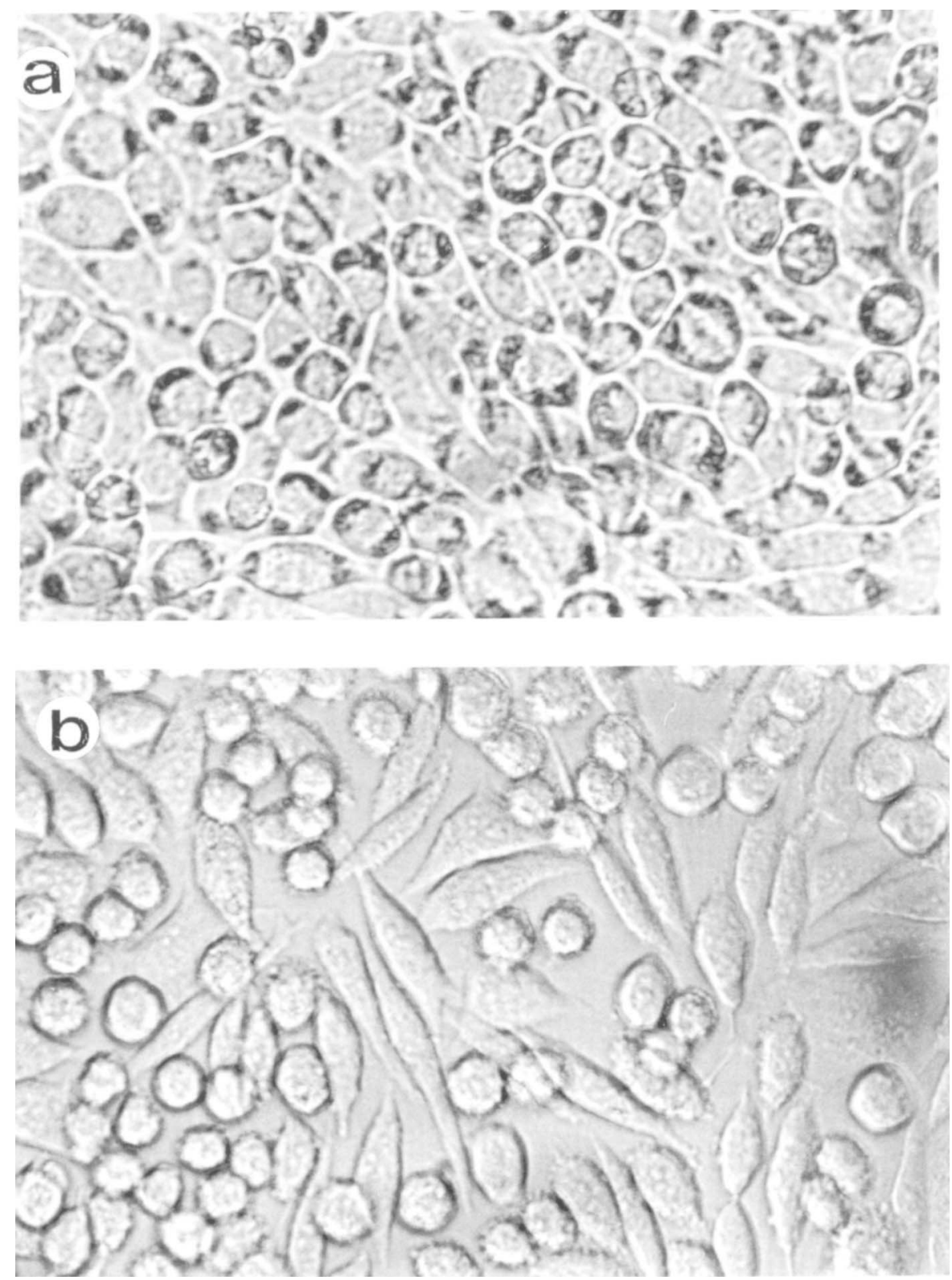

Fig. 5. a, The cytopathic effect (score of 3+, as described in Table 1) displayed by a monolayer of L-929 cells after exposure to $M$. fortuitum crude culture filtrate $\left(2 \mathrm{mg}\right.$ protein) at $37^{\circ} \mathrm{C}$ for $48 \mathrm{~h}(\times 1600)$. b, A monolayer of untreated $\mathrm{L}-929$ cells after incubation at $37^{\circ} \mathrm{C}$ for $48 \mathrm{~h}(\times 1600)$. (Olympus photomicrographic system, model PM-10-M, Japan.)

When Kreig et al. [2] originally described biological activity in the culture filtrates of $M$. ulcerans, they reported it to be a toxin unique to this organism. However, this study did not investigate the CCFs of other mycobacterial species for comparison. This work was performed in a subsequent investigation by Read et al. [3], where the activity of $M$. ulcerans culture filtrate was compared with those of $M$. marinum, $M$. chelonei and $M$. bovis. The results of this study confirmed those of the previous report, and further confirmed that cytoxic activity was confined to culture filtrates of $M$. ulcerans. However, the report also stated that $M$. marinum was observed to produce a positive CPE on L-929 cells, and M. chelonei was not tested in mouse footpads. Only the $M$. bovis strain BCG which was used showed no demonstrable biological activity in either the in-vivo or in-vitro assay. A more recent study aimed at comparing the lesions induced by $M$. ulcerans with those resulting from a spider bite used the method described by Kreig et al. [2] to produce culture filtrates [6]. This group also reported that they were unable to confirm the production of cutaneous ulcers as a result of exotoxin release from $M$. ulcerans.

The idea of a toxic substance being produced by $M$. ulcerans arose primarily because of the extensive necrosis associated with these infections. Read et al. [3] suggested that this necrosis was the consequence of a toxin, unique to M. ulcerans. However, the results of the present study suggest that a common mycobacterial antigen is responsible for the activity of culture filtrates seen in both cell culture and in the mouse footpad assay. Furthermore, an antiserum raised 


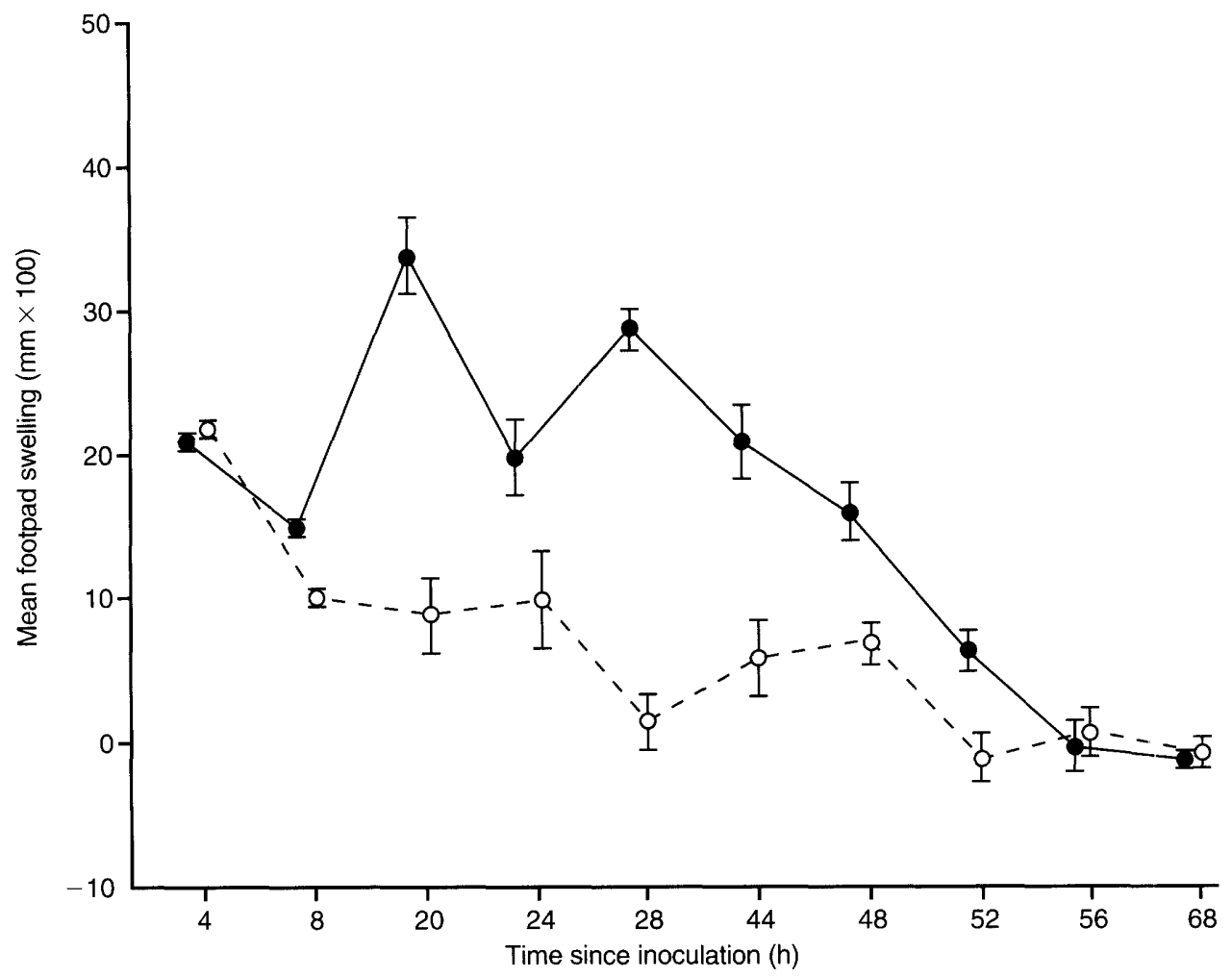

Fig. 6. Mean degree of mouse footpad swelling over time showing $95 \%$ confidence intervals, when injected with $0.025 \mathrm{ml}$ of mycobacterial crude culture filtrates: - $\mathrm{O}-$, Crude culture filtrates incubated at $37^{\circ} \mathrm{C}$ with antiserum raised against $M$. ulcerans crude culture filtrate; - - - untreated mycobacterial crude culture filtrates. Data are expressed as mean values of all mycobacterial species tested for each treatment.

Table 2. Results obtained after data from the toxin neutralisation assay at selected time points after inoculation were subjected to factorial ANOVAs

\begin{tabular}{llccrr}
\hline $\begin{array}{l}\text { Time } \\
(\mathrm{h})\end{array}$ & $\begin{array}{l}\text { Source of } \\
\text { variation }\end{array}$ & DFN & DFD & \multicolumn{1}{c}{ F } & \multicolumn{1}{c}{ p(F) } \\
\hline 4 & TRT & 1 & 20 & 0.16 & 0.693 \\
4 & ANT & 4 & 20 & 1.39 & 0.272 \\
4 & TRT by ANT & 4 & 20 & 0.20 & 0.937 \\
28 & TRT & 1 & 20 & 63.98 & $<0.001$ \\
28 & ANT & 4 & 20 & 1.77 & 0.174 \\
28 & TRT by ANT & 4 & 20 & 0.93 & 0.464 \\
68 & TRT & 1 & 20 & 0.02 & 0.896 \\
68 & ANT & 4 & 20 & 1.04 & 0.409 \\
68 & TRT by ANT & 4 & 20 & 0.82 & 0.526 \\
\hline
\end{tabular}

$\mathrm{DFN}$, degrees of freedom numerator; DFD, degrees of freedom denominator; TRT, treatment; ANT, antigen.

against M. ulcerans CCF was found to neutralise the biological activity of all mycobacterial CCFs tested. This suggests that the biological activity displayed by the CCF preparations may be due to the presence of either proteins or complex molecules of mycobacterial origin. As mycobacteria possess a unique cell wall, it is probable that the observed biological activity is the result of a cell wall component unique to mycobacter$\mathrm{ia}$, as these effects were not seen with E. coli CCF [2].

A recent study by Hayman [7] on the histopathology of $M$. ulcerans infection reported that the widespread necrosis observed in ulcerans disease represents infarction, rather than being a direct consequence of a cytotoxin. The latter report further describes the localised necrosis as being due to a deficient blood supply, resulting from intimal thickening and occlusion of small arteries. As yet, the mechanism that elicits this change is unknown, but it does not appear to be related to a bacterial toxin [7].

It is thus concluded that a diagnosis of ulcerans disease in man cannot be achieved from a serological assay based on a $M$. ulcerans toxin. Because of the high degree of antigenic similarity observed between mycobacterial species, it would be difficult to develop a serological assay with a sufficiently high degree of specificity. With the recent advent of DNA technology and gene cloning, a diagnostic assay involving the polymerase chain reaction (PCR) may provide more rewarding results.

This work was supported by a Merit Research Grant from James Cook University of North Queensland. We thank the technicians within the Department of Biomedical and Tropical Veterinary Sciences for their valuable assistance, Dr G. De'ath for statistical analysis and R. Cocciolone for support and advice along the way.

\section{References}

1. MacCallum P, Tolhurst JC, Buckle G, Sissons HA. A new mycobacterial infection in man. J Pathol Bacteriol 1948; 60: $93-122$.

2. Kreig RE, Hockmeyer WT, Connor DH. Toxin of Mycobacter- 
ium ulcerans. Production and effects in guinea pig skin. Arch Dermatol 1974; 110: 783-788.

3. Read JK, Heggie CM, Meyers WM, Connor DH. Cytotoxic activity of Mycobacterium ulcerans. Infect Immun 1974; 9: $1114-1122$

4. Hockmeyer WT, Krieg RE, Reich M, Johnson RD. Further characterisation of Mycobacterium ulcerans toxin. Infect Immun 1978; 21: $124-128$.
5. Laemmli UK. Cleavage of structural proteins during the assembly of the head of bacteriophage T4. Nature 1970; 227: $680-685$.

6. Atkinson RK, Farrell DJ, Leis AP. Evidence against the involvement of Mycobacterium ulcerans in most cases of necrotic arachnidism. Pathology 1995; 27: 53-57.

7. Hayman J. Out of Africa: observations on the histopathology of Mycobacterium ulcerans infection. J Clin Pathol 1993; 46: 5-9. 